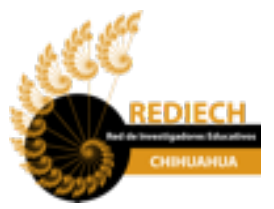

Red de Investigadores Educativos Chihuahua A.C. Chihuahua, México www.rediech.org

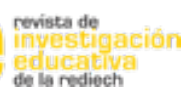

ISSN: 2007-4336

ISSN-e: 2448-8550

http://www.rediech.org/ojs/2017/index.php/ie rie rediech/index

Nancy Barragán Machado

Iram Isaí Evangelista Ávila

Paola Margarita Chaparro Medina

2019

\title{
Una interpretación desde la perspectiva de la hermenéutica analógica: la educación basada en la convivencia
}

IE Revista de Investigación Educativa de la REDIECH, 10(18), pp. 193-206. http://dx.doi.org/10.33010/ie_rie_rediech.v10i18.519

\section{(c) (1) (9)}

Esta obra está bajo licencia internacional

Creative Commons Reconocimiento-NoComercial 4.0. 


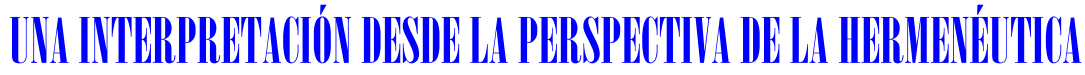

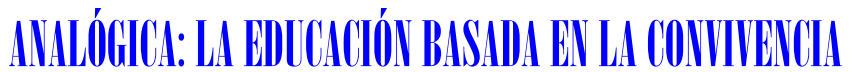

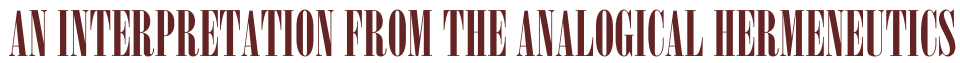

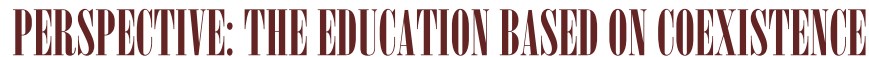

\author{
BARRAGÁN MACHADO Nancy \\ EVANGELISTA ÁVILA Iram Isaí \\ CHAPARRO MEDINA Paola Margarita
}

Regepción: Diciembre 15 de 2018 | Aprobado para publicación: MaRzo 29 de 2019

DOI: https://dx.doi.org/10.33010/ie_rie_rediech.v10il8.519

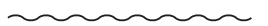

\section{Resumen}

El presente trabajo tiene como objetivo mostrar ciertos lineamientos de la educación basada en la convivencia y algunos de los problemas que se presentan cuando se intenta implementar en una cultura que privilegia la competencia. Como referencia se toma en cuenta el informe realizado a la UNESCO por la Comisión Internacional sobre la Educación para el Siglo XXI, presidida por Jacques Delors, en el cual se establecen los cuatro pilares de la educación, siendo de nuestro particular interés el pilar

Nancy Barragán Machado. Doctorado en Educación, Artes y Humanidades de la Facultad de Filosofía y Letras de la Universidad Autónoma de Chihuahua, México. Licenciada en derecho, especialista en gestión de conflictos y mediación, maestra en humanidades y certificada en solución de conflictos mediante técnicas de neuronegociación. Becaria del Consejo Nacional de Ciencia y Tecnología (Conacyt). Correo electrónico: nanbarragan@yahoo.com. ID: https:// orcid.org/0000-0002-3986-4717.

Iram Isaí Evangelista Ávila. Docente-investigador de tiempo completo en la Universidad Autónoma de Chihuahua, México. Es doctor en humanidades-literatura por la Universidad Autónoma Metropolitana y miembro del Sistema Nacional de Investigadores Nivel I. Su trabajo se centra en la teoría literaria aplicada a la literatura hispanoamericana, además de estudios inter y transdisciplinares. Tiene dos libros publicados: Ensayar al confabulador: acercamientos a la obra de Juan José Arreola y Hermenéutica de la autoficción. Ponente en congresos nacionales e internacionales de literatura contemporánea y humanidades. Perfil deseable Prodep. Correo electrónico: ievangelista@uach.mx. ID: https:// orcid.org/0000-0002-1065-082X.

Paola Margarita Chaparro Medina. Profesora-investigadora de la Facultad de Filosofía y Letras de la Universidad Autónoma de Chihuahua, México. Es doctora en filosofía con acentuación en estudios de la cultura por la Universidad Autónoma de Nuevo León. Tiene el reconocimiento al perfil Prodep. Entre sus publicaciones recientes se encuentra el libro Femina sacra, publicado por el ICM (2015), así como diversos artículos y capítulos de libros sobre temáticas en torno a los estudios de género y el pensamiento feminista. Actualmente se desempeña como secretaria de Investigación y Posgrado de la Facultad de Filosofía y Letras de la Universidad Autónoma de Chihuahua. Correo electrónico: pchaparro@uach.mx. ID: https://orcid.org/0000-0002-7270-9903. 
que se refiere a aprender a vivir juntos. Para este trabajo requerimos de la hermenéutica analógica como actitud y método, pues esta tiene en su esencia un camino intermedio que evita las interpretaciones absolutistas 0 relativistas. Esta exposición concluye en que una visión analógica permite otorgar claridad en cuanto a las necesidades que deben conciliarse en la educación: aprender habilidades técnicas para un desempeño en el mundo actual y aprender habilidades socioemocionales centradas en el trabajo en equipo, la cooperación, el respeto y el afecto por los demás.

\title{
Palabras clave: CONVIVENCIA ESCOLAR, HERMENÉUTICA ANALÓGICA, INFORME DELORS, PILARES DE LA EDUCACIÓN, EDUCACIÓN BASADA EN VALORES.
}

\begin{abstract}
The purpose of this document is to show certain guidelines of education based on coexistence and some of the problems that arise when trying to implement it in a culture that privileges competition. As a reference, it takes into account the report made to UNESCO by the International Commission on Education for the 21st Century, chaired by Jacques Delors, which establishes the four pillars of education, bringing to our attention the pillar about learning to live together. For this task, we require Analogical Hermeneutics as attitude and method, since it has in its essence an intermediate way that avoids absolutist or relativist interpretations. This analysis concludes that an analogical vision allows to grant clarity regarding the needs that must be reconciled in education: To learn technical skills to perform well in the current world; and, to learn social-emotional skills centered on teamwork, cooperation, respect and affection for others.
\end{abstract}

Keywords: SCHOOL COEXISTENCE, ANALOGIC HERMENEUTICS, DelorS REPORT, PILLARS OF EDUCATION, EDUCATION BASED ON VALUES.

\section{IVTronicoúis}

\section{El documento conocido como Informe Delors (1996) refiere que la} educación en la actualidad presenta diversos retos a superar, entre ellos: que las personas se conviertan en ciudadanos del mundo, pero sin perder las raíces de una cultura en particular; que exista una mundialización de la cultura, pero sin olvidar el carácter único de cada persona y su derecho a escoger su propio destino con base en el potencial que posee, así como que se atiendan las necesidades espirituales, pues la educación las ha relegado en privilegio de las necesidades materiales.

Con estos retos se pretende la concientización para valorizar aspectos éticos de la educación con el fin de que se pueda comprender al mundo desde una cierta unidad,

194 así como comprender al ser humano en su particularidad. Es decir, una armonización 
de los polos opuestos de la singularidad y la universalidad que se menciona en el informe (1996, p. 1):

Con esas perspectivas se ha vuelto imposible, y hasta inadecuado, responder de manera puramente cuantitativa a la insaciable demanda de educación, que entraña un bagaje escolar cada vez más voluminoso. Es que ya no basta con que cada individuo acumule al comienzo de su vida una reserva de conocimientos a la que podrá recurrir después sin límites. Sobre todo, debe estar en condiciones de aprovechar y utilizar durante toda la vida cada oportunidad que se le presente de actualizar, profundizar y enriquecer ese primer saber y de adaptarse a un mundo en permanente cambio.

Con estos planteamientos se destacan cuatro pilares de la educación: aprender a conocer, aprender a hacer, aprender a vivir juntos y aprender a ser.

De la revisión de la literatura se detectan dos posturas en cuanto a las implicaciones de este informe. Por un lado, existen argumentos que apelan a realizar cambios en la educación desde un paradigma de la colaboración y convivencia. Por otro lado, existe una postura crítica que nos hace ver un panorama real donde se dificulta seguir tales lineamientos a causa del paradigma imperante basado en la competencia. Ante tales visiones, es necesario un camino intermedio que nos otorgue una orientación y con el cual se eviten las interpretaciones unívocas o equívocas. La hermenéutica analógica es la idónea para ello, pues en sí misma tiene una esencia pacificadora al no destruir ni anular las posturas opuestas, sino al contrario, procura conciliarlas desde un juicio prudencial con el cual se pueda formar un camino. Este sentido que se busca es el que más se acerque hacia una interpretación significativa que permita el desarrollo socioemocional del ser humano:

[...] para hacer frente a los retos del siglo XXI, sería indispensable asignar nuevos objetivos a la educación y, por consiguiente, modificar la idea que nos hacemos de su utilidad. Una nueva concepción más amplia de la educación debería llevar a cada persona a descubrir, despertar e incrementar sus posibilidades creativas, actualizando así el tesoro escondido en cada uno de nosotros, lo cual supone trascender una visión puramente instrumental de la educación, percibida como la vía obligada para obtener determinados resultados (experiencia práctica, adquisición de capacidades diversas, fines de carácter económico), para considerar su función en toda su plenitud, a saber, la realización de la persona que, toda ella, aprender a ser [Delors, 1996, p. 1].

Creemos que lo anterior puede abonar al desarrollo del texto, ya que denuncia algunas carencias de los sistemas por competencias. A continuación, se desarrollará la idea por medio de un ejercicio de hermenéutica analógica.

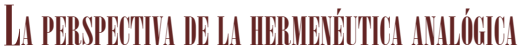

El filósofo mexicano Mauricio Beuchot es quien desarrolla este método de acercamiento. El autor resume la historia de la hermenéutica como aquella que se ha 
vertebrado a través de la pugna entre univocistas y equivocistas. Los primeros privilegian a la razón como base de la interpretación de los textos, la cual se caracteriza por buscar un sentido único y referencial; y los segundos buscan múltiples sentidos al texto, lo cual se ha asociado con la parte emocional y subjetiva, propiciando que todas las interpretaciones sean válidas (Beuchot, 2015). Una tercera postura es la hermenéutica analógica, la cual seguiremos en este trabajo, ya que busca un terreno medio entre estas aproximaciones y oscila hacia uno o hacia el otro según la necesidad. En esta postura predomina la diferencia sobre la semejanza. Es decir, hay más subjetividad que objetividad (Beuchot, 2005). La polarización la vemos entre las dos primeras categorías y el término medio en la tercera. La analogía recupera algo de la univocidad al intentar objetivar algo; por otro lado, recupera algo de la equivocidad al abrirse hacia la diferencia (Beuchot, 2009, 2011, 2014). ${ }^{1}$

Este método tiene dos aspectos: el de proporcionalidad y el de atribución. La proporcionalidad permite aglutinar o coordinar varias interpretaciones a través de un común denominador. La atribución busca distinguir, atender a las diferencias y aprovechar una estructura jerárquica para establecer analogados principales y secundarios. Es decir, permite establecer una interpretación como principal, en el sentido de que se considera más rica y más fecunda, sin pretender que agote toda la verdad textual, y las otras interpretaciones cercanas, que también son adecuadas, pero que se colocan en un orden jerárquico descendente (Beuchot, 2009, 2011, 2014).

Sobre esto, Evangelista (2014) otorga claridad sobre tres momentos del método analógico: el primer momento se refiere al análisis del texto y de otros textos relacionados, a lo cual se le llama subtilitas implicandi. El segundo momento es donde se explican los niveles semánticos de los textos en cuestión y se denomina subtilitas explicandi; para ese análisis tomaremos el siguiente apunte de Beuchot (2009, p. 23):

Subtilitas explicandi correspondiente a la semántica. Aquí se va al significado del texto mismo, pero no ya como sentido, si no como referencia, es decir, en su relación con los objetos, y por ello es donde se descubre cuál es el mundo del texto, esto es, se ve cuál es su referente, real o imaginario.

Y el último, el subtilitas applicandi se refiere al ejercicio donde el intérprete hace suyo lo que pudo ser la intención del autor, de tal forma que la función del investigador es lo que permite la conciliación entre el pensamiento del autor y el lector

${ }^{1}$ En el Tratado de hermenéutica analógica (2009), Beuchot menciona que el modelo positivista o univocista: "Sostiene que sólo hay una interpretación válida. Las demás son en su totalidad incorrectas" (pp. 40-41) y que el modelo romántico o equivocista "da predominio total a la diferencia, a esa diversidad de interpretaciones que introduce el yo irrepetible e intransferible, con el peligro de ser incomunicable" (p. 45). Por ello recomienda una hermenéutica analógica, la cual: "[...] las interpretaciones no fueran todas inconmensurables, equívocas, ni todas tuvieran que ser idénticas por sólo haber una posible, unívoca, sino que fueran en parte comunicables, precisamente por tener una parte de comunidad o igualdad, pero preponderantemente diversas por guardar en cierta medida la particularidad del intérprete" (p. 45). Debido a lo anterior, nos guiaremos en nuestro trabajo a través de la hermenéutica analógica; nos distanciamos, entonces, de una postura rígida e impositiva (unívoca) y evitaremos caer en juicios subjetivos y poco sustentables (equívoco); daremos prioridad al diálogo entre lo expuesto por autores reconocidos en el campo y junto con ellos expondremos nuestra interpretación. 
por aquello de la contienda entre lectura y significación (Evangelista, 2014). Con esta breve pincelada podemos entresacar algunos pasos metodológicos, los cuales se acomodan al propósito propio de este trabajo: ${ }^{2}$

1. En un primer momento se analiza el tema de la educación dentro del paradigma de la convivencia y otros textos relacionados. Esto se puede entender a razón de la subtilitas implicandi.

2. El segundo momento se refiere a analizar el contenido del tema que nos ocupa. Con ello se atiende a las significaciones, 0 en este caso al contenido del Informe Delors y el debate con la perspectiva analógica de su discurso, esto dentro de la categoría subtilitas explicandi.

3. El tercer momento se refiere a la contextualización analógica del tema, lo cual tiene paralelismo con la subtilitas applicandi, que se refiere al ejercicio donde el intérprete hace suyo lo que pudo ser la intención del autor. En este caso, lo que consideramos es el camino analógico de la educación basada en la colaboración y la convivencia.

La educación como ámbito de realidad es susceptible de ser conocida de diversas formas y cada una de ellas se aplica a la obtención del mejor conocimiento de la educación que es posible. Los principios de investigación pedagógica de objetividad, complejidad objetual, autonomía funcional, complementariedad metodológica y significación-validez avalan esta posición. La educación como ámbito de realidad es susceptible de ser contemplada como actividad y como ámbito de conocimiento; la educación como ámbito de realidad es una actividad cognoscible, enseñable, investigable y realizable [Touriñán, 2015, p. 3].

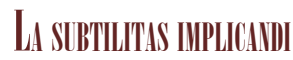

Los cuatro pilares planteados en el Informe Delors apuntan a una formación integral del ser humano para que se encamine hacia la comprensión de sí mismo, del otro y del mundo, lo cual va más allá de la adquisición de una competencia o de un oficio. Por eso, se dice que se trata de un nuevo paradigma, porque rompe con la visión tradicional de la educación, la cual, hay que recordar, se basa en que el docente es el centro de la dinámica enseñanza-aprendizaje y el papel del alumno se remite a solo escuchar y atender, sin ninguna interacción que lo estimule a participar dentro

${ }^{2}$ En Perfiles esenciales de la hermenéutica (2011), Beuchot menciona que el camino de la subtilitas se recoge desde: "La Edad Media se confirió a Juan Duns Escoto el apelativo de 'Doctor Subtilis', y esa sutileza consistía en encontrar siempre una posibilidad donde los otros no la veían; ellos solo veían dos" (41). Además, los términos: sutileza para entender o implicar, sutileza para explicar y sutileza para aplicar "aparecen ya en J.J. Rambach en Institutiones hermeneuticae sacrae, 1723 y reaparecen en Johann August Ernesti, Institutio Interpretis Novi Testamenti, 1761" (p. 42). Aquí el doctor Mauricio Beuchot retoma y además elabora una modificación, a subtilitas intelligendi, que es el primer momento: "[...] preferiría llamar subtilitas implicandi. La razón es que el significado sintáctico es el que se presupone en primer lugar; sin él no puede haber semántica ni pragmática" (p. 42). Así, Beuchot recoge esta tradición que causa analogía con lo expuesto por Gadamer en Verdad y método II y con la hermenéutica de Ricœur en Tiempo y narración; de esta manera, el autor pone el texto en su contexto, cumpliendo también con el método que propone. 
de dicha dinámica. Esta situación puso en tela de juicio la manera en que el estudiante afrontaba la adquisición de conocimientos, puesto que únicamente su acción se limitaba a repetir los patrones impuestos por el profesor. Así, estos pilares de la educación surgen como una forma de cambiar el proceso educativo y llevarlo paralelo con las exigencias del propio contexto.

El primer pilar se remite a aprender a conocer. Al llevarse a cabo, las posibilidades que ofrece la educación pueden aprovecharse a lo largo de la vida. El informe señala lo conveniente de estudiar aspectos generales de la cultura que sirvan de base para una educación permanente, es decir, que deje una huella dentro del estudiante y que el conocimiento no sea solamente pasajero, que perdure dentro de la persona con saberes útiles y prácticos, pero sin dejar de estudiar a fondo un número reducido de materias.

Este tipo de aprendizaje, que tiende menos a la adquisición de conocimientos clasificados y codificados que al dominio de los instrumentos mismos del saber, puede considerarse a la vez medio y finalidad de la vida humana. En cuanto a medio, consiste para cada persona en aprender a comprender el mundo que la rodea, al menos suficientemente para vivir con dignidad, desarrollar sus capacidades profesionales y comunicarse con los demás. Como fin, su justificación es el placer de comprender, conocer, de descubrir [Delors, 1996, p. 2].

El segundo pilar referido a aprender a hacer se expone a razón de la capacitación del estudiante en el uso de herramientas académicas que le ayuden en las situaciones sociales; hacer énfasis en socializar el conocimiento apoyado en el trabajo en equipo. Aprender a trabajar en conjunto fomenta otras capacidades, como la de saber argumentar, observar en diferentes perspectivas las problemáticas planteadas, entre otras.

Aprender a conocer y aprender a hacer son, en gran medida, indisociables. Pero lo segundo está más estrechamente vinculado a la cuestión de la forma profesional: ¿cómo enseñar al alumno a poner en práctica sus conocimientos y, al mismo tiempo, cómo adaptar la enseñanza al futuro mercado del trabajo, cuya evolución no es totalmente previsible? [Delors, 1996, p. 3].

El tercer pilar hace énfasis en el aprender a vivir juntos, y se refiere al aprendizaje de vivir con los demás desde la aceptación y la tolerancia. Este pilar es al que más énfasis le pone este informe, pues lo considera como el propulsor de un espíritu nuevo en la pedagogía, ya que la educación tiene la doble misión de enseñar desde la diversidad dentro del género humano, así como desde la toma de conciencia en cuanto a las semejanzas y la interdependencia que existe entre todos. Sin embargo, el informe reconoce que el descubrimiento del otro debe pasar de manera forzosa por el conocimiento de uno mismo, de donde se colige la gran tarea por parte de las instituciones socializantes -la escuela, la familia y la sociedad- de facilitarle la tarea al niño de descubrir quién es. De otra manera, concluye el informe, el individuo no podrá empatizar con los demás. 
Es conveniente en este punto dilucidar acerca de la educación basada en la convivencia, definir qué se entiende por convivir. Al respecto, Jares (2002, p. 9) explicó:

[...] convivir significa vivir unos con otros basándonos en unas determinadas relaciones sociales y en unos códigos valorativos, forzosamente subjetivos, en el marco de un contexto social determinado. Estos polos que marcan el tipo de convivencia están potencialmente cruzados por relaciones de conflicto, pero en modo alguno ello significa amenaza para la convivencia. Conflicto y convivencia son dos realidades sociales inherentes a toda forma de vida en sociedad.

Es decir, los elementos de una educación basada en la convivencia son: relaciones sociales, valores, contexto determinado y la existencia del conflicto en el devenir humano. Esto lo retomaremos más adelante.

El cuarto pilar consistente en aprender a ser, y se refiere al fortalecimiento del yo desde el reconocimiento de una doble acepción: la responsabilidad personal en el destino colectivo y la realización del potencial que cada persona tiene como ser particular:

[...] la educación debe contribuir al desarrollo global de cada persona: cuerpo y mente, inteligencia, sensibilidad, sentido estético, responsabilidad individual, espiritualidad. Todos los seres humanos deben estar en condiciones, en particular gracias a la educación recibida en su juventud, de dotarse de un pensamiento autónomo y crítico y de elaborar un juicio propio, para determinar por sí mismos qué deben hacer en las diferentes circunstancias de la vida [Delors, 1996, p. 8].

Como textos esclarecedores a este informe, tomamos como referencia a Cabello y Vázquez (2018), quienes establecen que las recomendaciones y retos del Informe Delors requieren el diseño e implementación de estrategias educativas contemporáneas que se adapten a las necesidades sociales de los nuevos modelos de familia. Para estos autores, la educación para la paz a que hace mención dicho informe, no quiere decir que se deban incluir temas o asignaturas en el currículo escolar sobre valores y principios, sino que se trata de un modelo de enseñanza fundamentado en una educación en valores, desde la influencia permanente de los tres agentes socializadores: familia, escuela y sociedad. La propuesta es la transversalidad en la educación que implica cambiar la enseñanza descriptiva por una enseñanza práctica. Esto requiere la interiorización de los valores en los estudiantes más que un aprendizaje basado en el análisis de estos. La interiorización de la paz es traducida como "un estado de bienestar que inicia con uno mismo y que se generaliza o se traspasa a la familia, la escuela y la comunidad" (p. 199). Con estas exposiciones, los autores proponen adicionar un quinto pilar al Informe Delors, el cual contemple: aprender a practicar.

También nos otorga claridad Jares (2001) al referir que la educación basada en la convivencia debe trabajar cuatro contenidos, tanto en el plano curricular como en las relaciones que se dan dentro de las escuelas:

1. La revisión del control y la disciplina. Este aspecto puede tener una lectura negativa, dice el autor, pero se debe tomar en cuenta que una opción que rechace 
todo tipo de control es ilusoria, pues se confunde obediencia con respeto. En realidad, se necesita rescatar el tema del respeto y una disciplina basada en la democracia, así como discutida y asumida por la comunidad estudiantil.

2. El cultivo de las relaciones interpersonales basadas en la afectividad. Para Jares, se trata de cambiar la cultura de la competitividad por una de tolerancia, reciprocidad y afirmación, retomando lo dicho por Paulo Freire en el sentido de que "la educación es un acto de amor" (p. 79).

3. La resolución positiva de los conflictos. Este aspecto se refiere a ver al conflicto como una parte inevitable de la vida, pero dándole un contenido de aprendizaje.

4. La prevención de la violencia. Este aspecto toma relevancia con el creciente problema del acoso escolar, o mejor conocido como bullying, el cual empieza a ser tema de interés para las investigaciones académicas por las alarmantes consecuencias socioemocionales.

Con esto podemos concluir que tanto el Informe Delors como los textos aludidos nos otorgan pautas para modelos educativos que se apeguen al paradigma de colaboración, los cuales deben incluir: calidad de procesos enseñanza-aprendizaje, calidad en estrategias de formación integral y calidad en las herramientas de preparación para la vida (Cabello y Vázquez, 2018, p. 117).

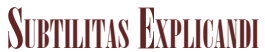

Hemos referido algunas implicaciones de la educación basada en la convivencia desde el Informe Delors y textos que se relacionan. Desde este enfoque, es compatible una educación en donde se aprenda un oficio con el cual se puedan cubrir las demandas del mundo actual competitivo; y, por otro lado, que se puedan aprender habilidades sociales de colaboración dentro del pilar de aprender a vivir juntos. Jares (2001, 2002, 2006) establece la necesidad de cambiar el paradigma de la educación competitiva por uno de convivencia, lo cual nos lleva a explorar diversas posturas críticas.

Maturana (2007) refiere que las generaciones anteriores estudiaban para devolver algo al país. Había una concepción de responsabilidad social en la construcción de este. Actualmente, el contexto empuja a los estudiantes a estudiar para competir dentro de un mercado profesional. El problema se ensancha al alentarse una educación basada en valores y principios del cooperativismo cuando el sistema educativo es parte también de una mentalidad global donde se espolea la competencia. Luego, Maturana sigue refiriendo que es prácticamente imposible educar bajo ciertas directrices si esto no es parte de un proyecto nacional. Lo anterior trae como consecuencia que el propósito individual y el propósito social no coincidan, porque las personas se preparan y estudian para entrar en la competencia profesional. Además, esto es consecuencia de una sociedad entera que avala la lucha y la competencia. El tema se vuelve más complejo cuando no se toma en cuenta que hay dos épocas cruciales para que los valores sean más aceptados: la infancia y la juventud. Al respecto, Maturana (2007, p. 11) dice que en la infancia se funda la posibilidad de que un ser humano pueda ser capaz de "aceptar y respetar al otro desde la aceptación y respeto de sí 200 mismo". En la juventud se prueba la validez de ese mundo de convivencia desde las 
coordenadas antes dichas. De poco sirve educar en valores y convivencia si el niño sale del aula y constata que ese discurso no está confirmado por la manera que vive su familia y comunidad, al igual el joven que prueba que tal educación es parte de una asignatura o materia educativa, pero no tiene nada que ver con el mundo de convivencia real. Es decir, aprende valores que debe respetar, pero "vive en un mundo adulto que las niega" (Maturana, 2007, p. 13).

Por su parte, Jares (2001, p. 81) señala las cuatro grandes consecuencias por el culto a la competitividad dentro del tema de la educación: "[...] reducción de la presencia del Estado [...]; la privatización de las funciones públicas [...]; desregulación de los controles públicos, pues se cree que el mercado lo hará [...]; y la promoción de la cultura empresarial como modelo a seguir [...]".

Para Maturana, la sana competencia no existe, porque la competencia se constituye en la negación del otro. Dentro de la biología no tiene cabida la competencia, porque los demás seres vivos no compiten, sino que esto es un fenómeno propiamente cultural y humano. Sin embargo, el lenguaje, de acuerdo con el autor chileno, se originó gracias a un modo de vida relacional entre los humanos al compartir alimentos, al placer de la convivencia, al encuentro sensual recurrente y toda una serie de coordinaciones consensuales. Por eso el autor sostiene que la emoción que hizo posible la hominización fue el amor. De ahí que muchas de las enfermedades humanas son originadas desde el desamor y estas se curan cuando se recupera el amor por sí mismo y por los otros $(2007,2013)$. Esto tiene relación con el tema que nos ocupa, porque, como lo señalamos con anterioridad, uno de los elementos de la convivencia son las relaciones sociales. Pero para Maturana solo se pueden llamar relaciones sociales las que se originan por la aceptación mutua entre los seres humanos y no por la competencia. En este sentido, no todas las relaciones que se dan en una comunidad estudiantil son sociales, porque se basan en el compromiso de cumplir cierto objetivo o tarea, pero no importa el aspecto de la aceptación de unos y otros.

Hemos abordado dos posturas opuestas: una constructiva o idealista, que señala que es posible el paradigma de la colaboración desde un mundo competitivo; y otra que refiere que no es posible. Por ello es necesario una postura matizada, como la que ofrece la hermenéutica analógica. Sin embargo, para llegar a esta postura intermedia es necesario traer el texto al contexto. Para esto tomamos en cuenta lo señalado por Maturana en el sentido de que la educación debe estar enmarcada dentro de un proyecto de nación. Para nuestro ejercicio, revisamos el proyecto de nación y algunas de las leyes educativas de México que competen a este trabajo.

En el Plan Nacional de Desarrollo 2013-2018 (Diario Oficial, 2013) se establece dentro de las cinco metas nacionales "una educación de calidad" (p. 9). En este apartado se señala que la educación debe impulsar "las competencias y las habilidades integrales de cada persona, al tiempo que inculque los valores por los cuales se defiende la dignidad personal y la de los otros" (p. 31). Con esto podemos concluir que se está tomando en cuenta al Informe Delors por lo que se refiere al reto de integrar singularidad y generalidad. Sin embargo, esta declaración se desdibuja cuando señala los tres ejes de la educación: 1) "Que los alumnos sean educados por los mejores maestros [...]"; 2) "Que la evaluación sea un instrumento para elevar la calidad de la enseñanza [...]"; y, 3) "Que la educación se convierta en responsabilidad 
compartida entre directivos, maestros, alumnos y padres de familia [...]" (p. 37). Además, se observa del contenido de todo este plan de desarrollo que hay un interés primordial en educar para hacerle frente a las demandas de un mundo globalizado. Es decir, hay más énfasis en la competitividad que en la colaboración.

Por otra parte, la reciente publicación del "Modelo educativo para la educación obligatoria reforma" (Diario Oficial, 2017) establece cinco grandes ejes: el planteamiento curricular, la escuela al centro del sistema educativo, formación y desarrollo profesional docente, inclusión y equidad y la gobernanza del sistema educativo.

En el eje del planteamiento curricular se establece la importancia de un enfoque humanista que tome en cuenta el desarrollo integral del estudiante, así como se incorporen al desarrollo académico las habilidades socioemocionales. Además, se establecen varios ámbitos que debe conocer el egresado: lenguaje y comunicación, pensamiento matemático, exploración y comprensión del mundo natural y social, pensamiento crítico y solución de problemas, habilidades socioemocionales y proyecto de vida, colaboración y trabajo en equipo, convivencia y ciudadanía, apreciación y expresión artística, atención al cuerpo y salud, cuidado del medio ambiente y habilidades digitales.

Como se puede observar, la educación basada en la convivencia sí se contempla entre los lineamientos de este nuevo modelo educativo. Incluso, en la exposición de motivos se indica que estos cambios responden a una exigencia social, porque el sistema educativo mexicano se ha caracterizado por una estructura vertical y rígida debido a la permanencia de la educación tradicional. También se puede constatar en el glosario que se toma en cuenta el Informe Delors y los cuatro pilares que ya hemos venido analizando a lo largo de este trabajo.

Por último, tenemos que el artículo séptimo de la Ley General de Educación de México (2018) señala que los fines de la educación, entre otros, son contribuir al desarrollo integral del individuo para que ejerza sus capacidades humanas; favorecer la adquisición de conocimientos y la capacidad de observación, análisis y reflexión críticos; promover el valor de la justicia, la cultura de la legalidad, la inclusión, la no discriminación, la paz y la no violencia, así como el respeto a los derechos humanos; fomentar actitudes solidarias y positivas hacia el trabajo, el ahorro y el bienestar general; y fomentar los valores y principios del cooperativismo. Ello nos lleva a concluir que dentro de esta ley sí está contemplada la educación basada en la convivencia y en los valores que enmarcan el buen vivir. En concreto, lo relativo al pilar que refiere el Informe Delors, de aprender a vivir juntos.

Ahora bien, este análisis del contexto también se explica en razón de los elementos que componen una educación basada en la convivencia, según Jares (2001): relaciones sociales, ciertos valores, contexto determinado y la existencia del conflicto en el devenir humano.

En cuanto a las relaciones sociales, ya dijimos que para Maturana no toda relación humana es social, pues para que sea social tiene que intervenir el amor o el afecto. En cuanto a los valores y al contexto determinado, de acuerdo con las leyes ya analizadas podemos afirmar que en México se enmarca la educación basada en la convivencia en los valores y principios del cooperativismo, así como en un enfoque 202 humanista que toma en cuenta el desarrollo integral del estudiante, que incluye además 
de lo académico, la parte socioemocional. De este contexto podemos señalar algunos aspectos: el proyecto de nación se encuentra desarticulado con las leyes educativas señaladas, pues el primero hace énfasis en la demanda del mercado global y las segundas pretenden cambiar el paradigma competitivo. Sin embargo, es de acotar que el modelo educativo es de recién publicación y aún no se observan sus efectos.

Por otro lado, el elemento que se refiere al conflicto como parte de la existencia humana, y que el estudiante debe aprender a vivirlo desde una perspectiva de aprendizaje, también nos lleva a una dimensión filosófica al preguntarnos para qué sirve la educación, de lo cual podemos encontrar múltiples respuestas. Del mismo Informe Delors se puede entresacar un sentido humano: que es el educar para que los seres humanos tengan un proyecto para toda la vida, y otra que se refiere a una educación en valores que podemos resumir como aquel que está basado en la colaboración y el afecto por el otro.

\section{SIITTIIIIIS IPPIIIIIIIII}

Los apartados anteriores hacen referencia a los aspectos del Informe Delors que nos encaminan hacia el sentido buscado. Ahora es necesario ahondar, desde nuestra perspectiva, sobre dicho sentido. Con esto cubrimos el tercer paso metodológico que consiste en hacer nuestro el texto. Al hacerlo, mostramos la intencionalidad del texto y nuestra propia intencionalidad. También hacemos uso de la facultad humana analógica, de la cual Beuchot señala como aquella que:

[...] pone en movimiento la pulsión analógica en el hombre, debido a la cual el ser humano utiliza una voluntad de poder en el uso de la analogía. Esta voluntad nos proyecta hacia más allá del texto, y nos empuja hacia la intencionalidad del autor, a pesar de que se nos impone nuestra propia intencionalidad de lectores o intérpretes [Beuchot, 2015b].

La doble misión que tiene la educación, de acuerdo con el Informe Delors, es educar en la diversidad humana y en la toma de conciencia en cuanto a las semejanzas y la interdependencia que existe entre todos los seres humanos. Con esto se deja en evidencia que no basta la acumulación de conocimientos, sino que es necesario voltear a las relaciones sociales basadas en el afecto y la convivencia. Estos retos colocan a las instituciones socializantes, como son la escuela, la familia y la sociedad, de inculcarle al niño, y al estudiante en general, la tarea de descubrirse a sí mismo, así como descubrir la riqueza de las relaciones sociales en un mundo que es cada vez más complejo y que desafortunadamente se caracteriza por una cultura de competencia.

Ahora bien, ya hemos trazado las diferencias entre visiones constructivas y las visiones críticas a través de una muestra representativa, pues no es posible abarcarlas todas. Las primeras nos señalan los fines y se colocan en la postura idealista. Las segundas problematizan los medios que se tienen para llegar a los fines y se ubican dentro de una postura crítica del contexto. Estas visiones son complementarias, porque necesitamos comprender los medios para alcanzar los objetivos. Por otro lado, en el terreno de las semejanzas, ambas nos acucian a replantear la educación, 
porque algo definitivamente no está marchando en dirección hacia aquello que hemos considerado dentro de esta exposición como una mejora dentro de la impartición de la educación. Esto nos coloca en el horizonte de la ética pues, aunque este tema es amplio para abordarlo en este trabajo, es importante mencionarlo. Esta ética la consideramos desde su raíz original en griego, ethos, que se puede entender de dos maneras: ethos, como morada universal, abrigo permanente tanto de animales como de seres humanos, y de manera particular como la "morada del ser humano que le permite sentirse bien en el mundo" (Boff, 2001, p. 26). En este sentido, la crisis planetaria que incluye violencia, desigualdad social y degradación ambiental permite cuestionarnos si estamos haciendo las cosas de la manera correcta para sentirnos bien en el mundo. El hecho de que se alcen voces para poner en el centro la problemática del ser humano, nos indica que hay un síntoma que se hace visible en todas las áreas de actividad del hombre y la mujer, lo que incluye la educación por ser una de las vías socializantes por excelencia. Este síntoma se percibe como si ya no nos sintiéramos bien en nuestra casa planetaria por la manera en que nos estamos relacionando entre humanos, con otras especies y con el planeta Tierra. La educación se presenta como el camino para cambiar de paradigmas destructivos, pues la enfermedad y el remedio están en el propio ser humano. Por eso, la importancia de no ver el planteamiento del Informe Delors como algo aislado, sino como algo enraizado en esa ética que nos apela a transformar la morada en un mejor lugar. Esto nos habla de un aspecto universal, pero también de un aspecto particular, tal y como lo señala el Informe Delors en cuanto al reto de unificar lo general con lo singular.

Este aspecto particular se relaciona con el problema añejo del hombre que busca un sentido para su vida desde una doble acepción: dirección y significado, tanto un proyecto de vida como una significación que dé calidad a su existencia. El sentido objetivo es aquello en lo que encontramos la perfección, y el subjetivo o personal es la felicidad (Beuchot, 2011b). Para este autor, la virtud principal es la del amor, y su ejercicio repercute en la felicidad. Todo lo que avance en esa dirección dará sentido. De donde se colige entonces un ejercicio analógico al tomar en cuenta el aspecto de educar en herramientas que le otorguen al ser humano un proyecto de vida, como en este caso es el desempeño profesional, y por otro lado la parte afectiva que es necesaria para lograr la felicidad del hombre desde lo subjetivo. La hermenéutica analógica, al privilegiar el sentido entre las dos posturas opuestas, nos muestra que no debemos olvidar que la educación no es un bien de consumo, a lo cual nos parece adecuado darle un analogado principal con la educación basada en la convivencia, y después a las técnicas profesionales que se despliegan en un mundo competitivo. Bajo nuestro enfoque es preferible aprender a ser y a aprender a vivir juntos, pues si las relaciones sociales se pierden, de nada sirve que aprendamos a aprender 0 aprendamos a hacer. En otras palabras, si perdemos el sentirnos bien en nuestra propia morada humana, no bastará la acumulación de conocimientos.

En resumen, podemos decir que la hermenéutica analógica ayuda a conciliar los polos entre las visiones constructivas y las visiones críticas, ya que de otra manera no podemos visualizar el punto donde nos encontramos y hacia dónde queremos llegar. Se trata entonces de una doble lectura, como señala Jares (2001): una en positivo, 204 que permite afirmar y divulgar; y otra en negativo, donde se trata de cuestionar. De 
tal manera que el Informe Delors tiene una relevancia cuando lo miramos desde una perspectiva más amplia al instalarlo desde el horizonte de la ética y el significado de la vida.

\section{Covculsiones}

La manera de proceder de la hermenéutica analógica, a través de los tres momentos de la subtilitas, permite realizar una interpretación intermedia entre dos posturas: la constructiva, que señala la posibilidad de una educación basada en la convivencia, y la crítica, que cuestiona su factibilidad en un mundo competitivo. Para matizar fue necesario exponer varias ideas basadas en el amor o en el afecto que dan origen a las relaciones sociales, así como llevarlas a un contexto en específico, como es el caso de México. Se concluye que una educación basada en la convivencia tiene que seguir fomentándose a pesar de que vivamos en un mundo que fomenta la competencia. Sin embargo, desde una visión analógica se trata de una inversión en valores, donde la colaboración y el afecto se presentan como el analogado principal, y las competencias profesionales como el analogado secundario. Esto implica complementar el lado metafórico y el lado metonímico de la existencia humana, que están en constante tensión. El lado metafórico son los ideales a los que apunta el Informe Delors, y el lado metonímico es la realidad del contexto. Es necesario entonces comprender primero el contexto para hacer los ajustes necesarios para llegar a los ideales de una educación basada en la convivencia.

\section{Rererencus}

Beuchot, M. (2005). En el camino de la hermenéutica analógica. Salamanca, España: Editorial San Esteban.

Beuchot, M. (2007). Hermenéutica analógica, símbolo, mito y filosofía. México: Universidad Autónoma de México.

Beuchot, M. (2009). Tratado de hermenéutica analógica. Hacia un nuevo modelo de interpretación. México: Editorial Ítaca.

Beuchot, M. (2011). Perfiles esenciales de la hermenéutica. México: Fondo de Cultura Económica.

Beuchot, M. (2011b). Sobre el sentido de la vida, desde una hermenéutica analógica. Notandum, 25(I). Recuperado de: http://www.hottopos.com/notand25/09-16Beuchot.pdf

Beuchot, M. (2014). La huella analógica del caminar humano. Madrid, España: Editorial Acci.

Beuchot, M. (2015). Elementos esenciales de una hermenéutica analógica. Diánoia, 60(74), 127-144. Recuperado de http://www.scielo.org.mx/scielo.php?script=sci isoref\&pid=S018524502015000100006\&lng=es\&tlng=es

Beuchot, M. (2015b). Potencialidad y vigencia de una hermenéutica analógica. Conhecimento \& Diversidade, Niterói, 13(2). Recuperado de https//revistas.unilasalle.edu.br/index.php/ conhecimento diversidade/article/viewFile/1797/1420

Boff, L. (2001). Ética planetaria desde el Gran Sur. Madrid, España: Editorial Trotta.

Cabello, P. y Vázquez, R. (2018). Cultura y educación para la paz. Una perspectiva transversal. Ciudad de México, México: Tirant lo Blanch. 
Delors, J. (1996). La educación encierra un tesoro. Informe a la Unesco de la Comisión Internacional sobre la Educación para el Siglo XXI. Madrid, España: Ediciones Santillana.

Diario Oficial de la Federación. (2013, mayo 20). Plan Nacional de Desarrollo 2013-2018. Recuperado de http://www.cofemer.gob.mx/documentos/marcojuridico/rev2016/PND\%20 2013-2018.pdf

Diario Oficial de la Federación. (2017, junio 28). Modelo educativo para la educación obligatoria. Recuperado de http://www.dof.gob.mx/nota_detalle. php? $\operatorname{codig} 0=5488338 \&$ fecha $=28 / 06 / 2017$

Evangelista, I. (2014). Aproximaciones a dos cuentos de Juan José Arreola a través de la hermenéutica analógica. Hermes Analógica, (5), 17-33. Recuperado de https://sites.google. com/site/hermesanalogica/no-5---2014

Jares, X. (2001). Educar para la paz y la convivencia: tarea de todas y todos. Coruña, España: Facultad de Ciencias de la Educación de la Universidad de A. Coruña. Recuperado de http://redined.mecd.gob.es/xmlui/bitstream/handle/11162/6910/12040003REV.pdf? sequence $=1$ \&isAllowed $=\mathrm{y}$

Jares, X. (2002). Aprender a convivir. Revista Interuniversitaria de Formación del Profesorado, (44), 79-92. Recuperado de http://www.redalyc.org/articulo.oa?id=27404405

Jares, X. (2006). Pedagogía de la convivencia. Barcelona, España: Editorial Graó.

Ley General de Educación (2018). Recuperado de https://www.sep. gob.mx/work/models/sep1/ Resource/558c2c24-0b12-4676-ad90-8ab78086b184/ley_general_educacion.pdf

Maturana, H. (2003). Amor y juego, fundamentos olvidados de lo humano desde el patriarcado a la democracia (6a. ed.). Chile: Lom Ediciones.

Maturana, H. (2007). Emociones y lenguaje en educación y politica. Chile: Comunicaciones Noreste.

Touriñán, J. (2015). La complementariedad metodológica: principio de investigación pedagógica. Recuperado de https://sites.google.com/site/hermesanalogica/no-6---2015 